Revista Colombiana de Obstetricia y Ginecología Vol. 61 No. 1 • 2010 •(42-48)

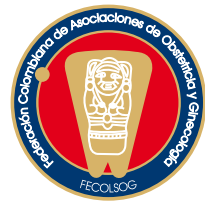

INVESTIGACIÓN ORIGINAL

\title{
SEGURIDAD Y ACTIVIDAD DE LA SOLUCIÓN DE BARDANA COMO COADYUVANTE EN EL MANEJO DEL PRURITO VULVOVAGINAL EN COLOMBIA. ENSAYO CLÍNICO FASE II
}

\section{A phase III clinical trial of burdock solution as an adjuvant in managing vulvovaginal pruritus in Colombia.}

\author{
Hoover Canaval, M.D. *, Jairo Bonilla, M.D.**, Claudia Scarpetta, M.D.***, \\ Fernando Martínez, M.D.***, Jaime Orejarena, M.D.***, Diana Solórzano, M.D.***, \\ Colaboradores $* * * *$ \\ Recibido: julio 23/09 - Aceptado: marzo 8/10
}

\section{RESUMEN}

El prurito genital puede ser la manifestación de problemas alérgicos, infecciones genitales, entidades sistémicas o una reacción primaria. El manejo de este síndrome es un reto para ginecólogos y médicos generales.

Objetivo: evaluar la seguridad y actividad de la solución de bardana al 1,2\% en aplicaciones externas diarias por 5 días, como coadyuvante en el manejo de las vulvovaginitis para generar alivio del prurito.

Metodología: el presente es un ensayo clínico de fase II, multicéntrico, abierto, no comparativo, realizado en pacientes con prurito genital, quienes fueron evaluadas en 14 ciudades colombianas. En el

* Profesor Asistente, Departamento de Ginecología y Obstetricia, Facultad de Salud, Universidad del Valle. Hospital Universitario del Valle "Evaristo García” E.S.E. Calle $5^{\mathrm{a}} \mathrm{N}^{\circ} 36-08$, piso $6^{\circ}$. Cali (Colombia). Correo electrónico: hcanaval@univalle.edu.co

** Profesor del Departamento de Ginecología y Obstetricia, Fundación Universitaria de Ciencias de la Salud. Bogotá (Colombia).

*** Médicos Gineco-obstetras de Armenia, Barranquilla, Bucaramanga, Bogotá, Cali, Cúcuta, Ibagué, Medellín, Santa Marta, Sincelejo o Valledupar (Colombia).

***** Lista completa al final del documento. estudio se incluyeron a todas aquellas mujeres mayores de 18 años con prurito genital sin importar su etiología, quienes no hubiesen recibido tratamiento alguno para la vulvovaginitis o la vulvovaginosis en los 30 días previos a su inclusión en el estudio y quienes, además, no hubiesen utilizado ningún producto farmacéutico de manera tópica en los 8 días anteriores al inicio del tratamiento. Asimismo, se excluyeron todas las pacientes en estado de embarazo, con trastornos psíquicos o mentales de difícil control y con antecedentes de alergias a medicamentos. Se evaluó la edad de la paciente, la intensidad del prurito, la presencia de irritación vulvar, leucorrea, los agentes infecciosos asociados con ésta y la tolerancia.

Resultados: se incluyeron 154 pacientes de las cuales se excluyeron 2 por no asistir al último control. Al final de los tratamientos, $83,6 \%$ de ellas manifestaban no tener prurito o sólo en una intensidad mínima y 11,8\% lo referían como leve. El prurito clasificado inicialmente en 30,5\% de las mujeres como severo se encontró sólo en 2,6\% al segundo día y tan sólo en 1,3\% persistía al final de 
la terapia. Similares resultados se obtuvieron en los subgrupos con infección demostrada por sólo cándida o sólo bacterias.

Conclusión: la utilización local complementaria de una solución tópica de bardana en mujeres con prurito genital podría ser una alternativa eficaz de manejo.

Palabras clave: prurito, solución, candidiasis, vulvovaginal, eczema, eritema, leucorrea, vulvar.

\section{SUMMARY}

Genital itching is associated with allergic and infectious aetiologies, systemic causes or a primary reaction. Managing this syndrome is a challenge for gynaecologists and general practitioners.

Objective: evaluating the safety and activity of a $1.2 \%$ burdock solution in external applications per day, for 5 days, as an adjuvant in managing vulvovaginitis to relieve itching.

Methodology: this was an open, non-comparative, multicentre, phase II clinical trial involving patients suffering from genital pruritus. The evaluation was made in 14 Colombian cities; it included women aged over 18 suffering from genital itching (regardless of aetiology) who had been not been treated for vulvovaginitis or vulvovaginosis during the 30 days prior to their inclusion in the study and had not used any topical pharmaceutical product during the 8 days before beginning treatment recommended by the study. Patients were excluded who were suffering from psychiatric or mental disorders, difficult to control, pregnant or had a history of drug allergy. The patients' ages, itching intensity, presence of leukorrhoea (vulvar irritation) and associated infectious agents were classified.

Results: 152 patients were included in the study after fulfilling the inclusion criteria; 2 of them were excluded for not attending the last control at the end of the treatment. $83.6 \%$ reported no itching by this time, or only minimum intensity, and $11.8 \%$ regarded it as just being slight. Itching initially classified by $30.5 \%$ of the patients as being severe at the start was only rated so by $2.6 \%$ on the second day; $1.3 \%$ still rated it as being severe at the end of therapy. Similar results were obtained in the subgroups suffering from single candida infection or single bacteria.

Conclusion: the supplementary local use of a $2 \%$ topical burdock solution in women suffering from genital itching could be an effective management alternative.

Key words: pruritus, burdock solution, candidiasis, vulvovaginal, eczema, erythema, vaginal discharge, vulvar.

\section{INTRODUCCIÓN}

El prurito es una sensación cutánea que genera un deseo de rascarse la parte afectada, ${ }^{1}$ lo cual aumenta a su vez la sensación de prurito, creándose con frecuencia un círculo vicioso. El prurito se ha asociado con múltiples etiologías alérgicas, infecciosas, otras de origen sistémico o como una reacción secundaria. ${ }^{1}$ La investigación sobre los mecanismos del prurito se ha apoyado en los estudios desarrollados en el dolor, puesto que estos dos síntomas comparten fenómenos moleculares y neurofisiológicos comunes. Esto quiere decir que tanto las sensaciones de dolor como de comezón, se originan en una red de terminaciones nerviosas libres en la unión dermoepidérmica, cuya activación puede ser originada por un estímulo térmico, químico, eléctrico o mecánico, ya sea interno o externo. ${ }^{1-3}$

Por otra parte, el prurito vulvovaginal no debe considerarse como una enfermedad sino como un síntoma y, como tal, debe procurarse obtener un diagnóstico etiológico que permita el enfoque terapéutico más adecuado. Dentro de las causas de prurito o de picor genital, con mucha frecuencia se encuentran en la práctica médica diaria, las infecciones de la vulva y la vagina, estando la infección por cándidas en 40\% a 50\%, seguida de la vaginosis bacteriana en $20 \%$ a 25\% y, finalmente, la tricomoniasis vaginal en $5 \%$ a $15 \% .{ }^{4,5}$ La etiología bacteriana del prurito es motivo de controversia, ya que algunas bacterias implicadas como productoras de infecciones pueden estar formando parte de la flora normal de la vulva y la vagina en mujeres 
sexualmente activas. ${ }^{4,5}$ Todas estas infecciones se enmarcan en cuadros conocidos como vulvovaginitis o vulvovaginosis, definidas como uno de los motivos más frecuentes de consulta en ginecología. ${ }^{4}$

Debido a lo anterior, no existe una terapia universal para el prurito, por lo que debe siempre buscarse la etiología. ${ }^{5}$ Sin embargo, las causas de prurito son tan variadas que en muchas ocasiones mientras se está realizando el estudio etiopatogéni$\mathrm{co}$, los pacientes requieren un tratamiento exclusivo y directo del mismo ya que puede ser un síntoma tan intenso que hasta resulte incapacitante y tarde varios días en ceder mientras la terapia etiológica inicia su acción. ${ }^{5}$

Farmacológicamente se han utilizado preparaciones tópicas a base de ácido láctico o acético, corticoides y antihistamínicos administrados tanto local como sistémicamente, con lo que frecuentemente se obtienen resultados variables de baja efectividad. ${ }^{1,4}$

Ante lo anterior, la solución de bardana al 1,2\% surge como una opción terapéutica interesante e importante para el control del prurito vulvar. ${ }^{5}$ Ésta es una solución estandarizada que contiene como principio activo los extractos hidroglicólicos de las hojas (folium bardanae) y las raíces (radix bardanae) de la bardana al 1,2\%. Esta solución se ha utilizado como coadyuvante en el manejo de la vulvovaginosis de cualquier etiología. ${ }^{3}$ Igualmente, se ha considerado que los principios activos poseen acciones neutralizadoras de las terminales nerviosas cutáneas en piel y mucosas, además de modificar el $\mathrm{pH}$ que limita el crecimiento de hongos oportunistas en la vulva y el periné. ${ }^{6}$

Por lo tanto, el objetivo de este estudio es evaluar la seguridad y la actividad de la utilización de una solución de bardana al 1,2\% como coadyuvante importante en el manejo del prurito vulvovaginal de diversas etiologías.

\section{METODOLOGÍA}

\section{Diseño}

El presente estudio es un ensayo clínico de fase II, multicéntrico, abierto, no comparativo, llevado a cabo entre los meses de agosto y octubre de 2008 en 14 ciudades de Colombia. Se incluyeron a todas aquellas pacientes mayores de 18 años que no hubiesen recibido tratamiento sistémico alguno para la vulvovaginitis o la vulvovaginosis en los 30 días anteriores a su inclusión en el estudio y quienes, además, no hubiesen utilizado ningún producto farmacéutico de manera tópica en los 8 días anteriores al inicio del tratamiento. Es importante aclarar que esas mujeres aceptaron participar en el estudio por medio de la firma de un consentimiento informado. En contraste, se excluyeron a las pacientes con trastornos psíquicos o mentales, aquellas que tuviesen impedimento para suministrar la información requerida, quienes presentaban menstruación o sangrado vaginal que ameritaba el uso regular de "toallas sanitarias", pacientes de difícil seguimiento, en embarazo y mujeres con historia clara de reacciones alérgicas medicamentosas. Se hizo un muestreo secuencial por conveniencia.

Durante la primera visita a la institución médica, el médico indagó y cuantificó la intensidad del prurito genital, asignando una calificación de 0 a 10 en una escala análoga. Se calificó el prurito de 0 a 2 puntos cuando lo referían como de intensidad mínima, de 3 a 5 puntos como intensidad leve, de 6 a 8 puntos el de intensidad moderada y de 9 a 10 puntos si lo describían como de intensidad severa.

También en esta primera visita y, de manera simultánea, se evaluaron los grados de irritación vulvovaginal, determinados por irritación local, eritema, úlceras o laceraciones vulvares. En el mismo momento se realizó la toma de una muestra para el análisis microbiológico del frotis vaginal, incluyendo el examen microscópico en fresco y la coloración de Gram, antes de iniciar el tratamiento y sólo cuando el cuadro clínico no daba una muy alta probabilidad de claridad etiológica sindrómica.

Posteriormente, las pacientes recibieron instrucciones de manejo que incluían una aplicación cada 12 horas de la solución de bardana al 1,2\% por 5 días continuos. Fue igualmente considerado el iniciar un plan terapéutico específico para el manejo etiológico respectivo, cuando estuvo indicado. 
Todas las pacientes recibieron una llamada telefónica como medida de control al segundo día de iniciado el tratamiento, donde se evaluó nuevamente la intensidad del prurito genital utilizando el instrumento respectivo, el cual fue llenado directamente por el médico tratante a través de la información proporcionada por vía telefónica y, de igual manera, por la paciente en su casa. Finalmente, durante la tercera entrevista efectuada como consulta médica formal, al $6^{\circ}$ día, un día después de terminar la medicación, se evaluó la irritación vulvar y la intensidad del prurito. Adicionalmente, en esta última visita, se utilizó una escala de tolerabilidad que determinaba 4 ítems a saber: excelente, buena, regular o mala.

\section{Variables a medir}

De otro lado, durante este estudio, se midieron la edad de la paciente, la intensidad del prurito genital, la presencia de irritación vulvar y la presencia de síntomas como: leucorrea, diagnóstico etiológico asociado y tolerabilidad. Los resultados se presentaron mediante proporciones para variables categóricas.

\section{RESULTADOS}

Se incluyeron 154 pacientes de las cuales se excluyeron 2 por no asistir al último control. Las pacientes incluidas oscilaban entre los 19 y 64 años, 32\% tenían entre 18 y 30 años, 36\% entre 31 y 40 años y 32\% eran mayores de 40 años. Todas procedían del área urbana y $62 \%$ de ellas presentaron leucorrea asociada y $75 \%$ eritema vulvar.
La calificación inicial del prurito y la dada durante el seguimiento al segundo y sexto día se presentan en la tabla 1. Entre los principales resultados se vislumbró una mejoría notable para todos los grupos de pacientes en relación con la intensidad del síntoma primario, el prurito, observándose al final de los tratamientos que $83,6 \%$ de las pacientes manifestaban no tener prurito y $11,8 \%$ de ellas ya sólo lo referían como leve o de intensidad mínima. Cabe destacar que esta mejoría fue notoria desde el segundo día del tratamiento.

Por otra parte, se observó un importante cambio en la evolución de otros síntomas y signos asociados (figura 1), y se dispuso del análisis microbiológico en 113 de las 152 pacientes, cuyos resultados se muestran en la figura 2.

Además, 87,9\% del grupo de pacientes que al ingreso presentaban Cándida albicans $(\mathrm{n}=37)$ como único agente patógeno clasificaron el prurito como de intensidad severa o moderada. Al segundo día de utilización de la solución de bardana al 1,2\%, sólo 27,3\% manifestaban este mismo grado de severidad, momento en el que apenas iniciaban la terapia antimicótica. Al final del estudio ninguna paciente experimentaba el síntoma como severo, apenas 3\% de ellas lo referían como moderado y 84,8\% tenía ausencia o presentaban únicamente una sensación mínima del prurito.

Del mismo modo, de aquellas pacientes con frotis vaginal que mostraban presencia sólo de bacterias $(n=30)$ como agentes patógenos, 60\%

\section{Tabla 1. Intensidad del prurito vulvovaginal al inicio y al final del seguimiento.}

\begin{tabular}{|c|c|c|c|c|c|c|}
\hline \multirow[t]{2}{*}{$N=152$} & \multicolumn{2}{|c|}{ Primera visita } & \multicolumn{2}{|c|}{$\begin{array}{l}\text { Segundo día de tratamiento } \\
\text { (Contacto telefónico) }\end{array}$} & \multicolumn{2}{|c|}{$\begin{array}{l}\text { Quinto día de tratamiento } \\
\text { (Última visita) }\end{array}$} \\
\hline & $\mathbf{N}^{\circ}$ & $\%$ & $\mathbf{N}^{\circ}$ & $\%$ & $\mathbf{N}^{\circ}$ & $\%$ \\
\hline Severo & 55 & 36,2 & 4 & 2,60 & 2 & 1,3 \\
\hline Moderado & 78 & 51,3 & 29 & 19,1 & 4 & 2,6 \\
\hline Leve o mínimo & 19 & 12,5 & 62 & 40,8 & 18 & 11,8 \\
\hline Ausente & 0 & 0 & 57 & 37,5 & 127 & 83,6 \\
\hline NS/NR* & 0 & 0 & 0 & 0 & 1 & 0,7 \\
\hline
\end{tabular}

*NS/NR: no sabe/no responde. 
Figura 1. Evolución de signos y síntomas a la primera visita médica y después del $5^{\circ}$ día de tratamiento.

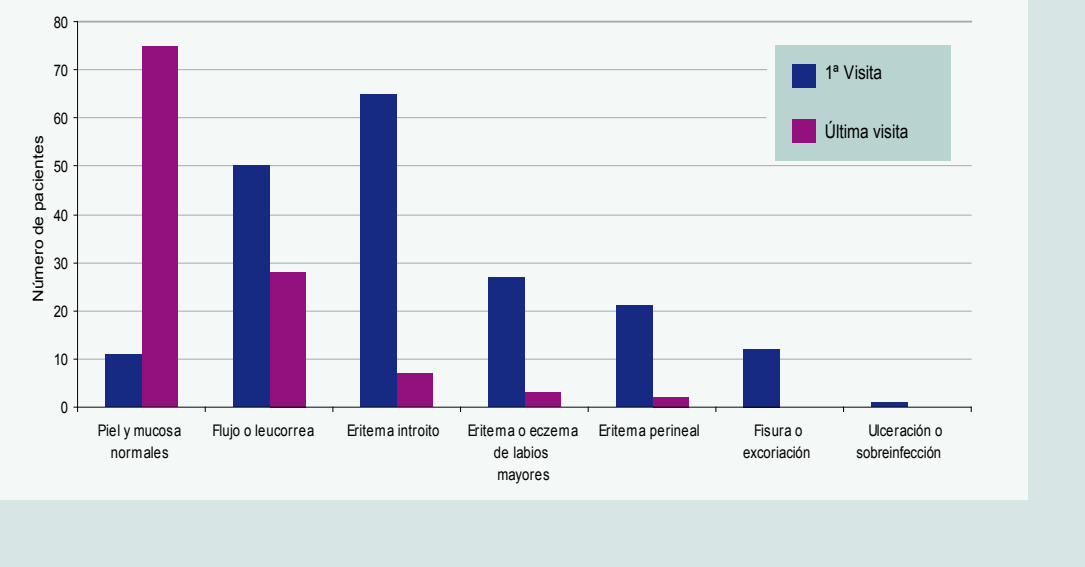

Figura 2. Resultado del estudio microbiológico de secreción vaginal.

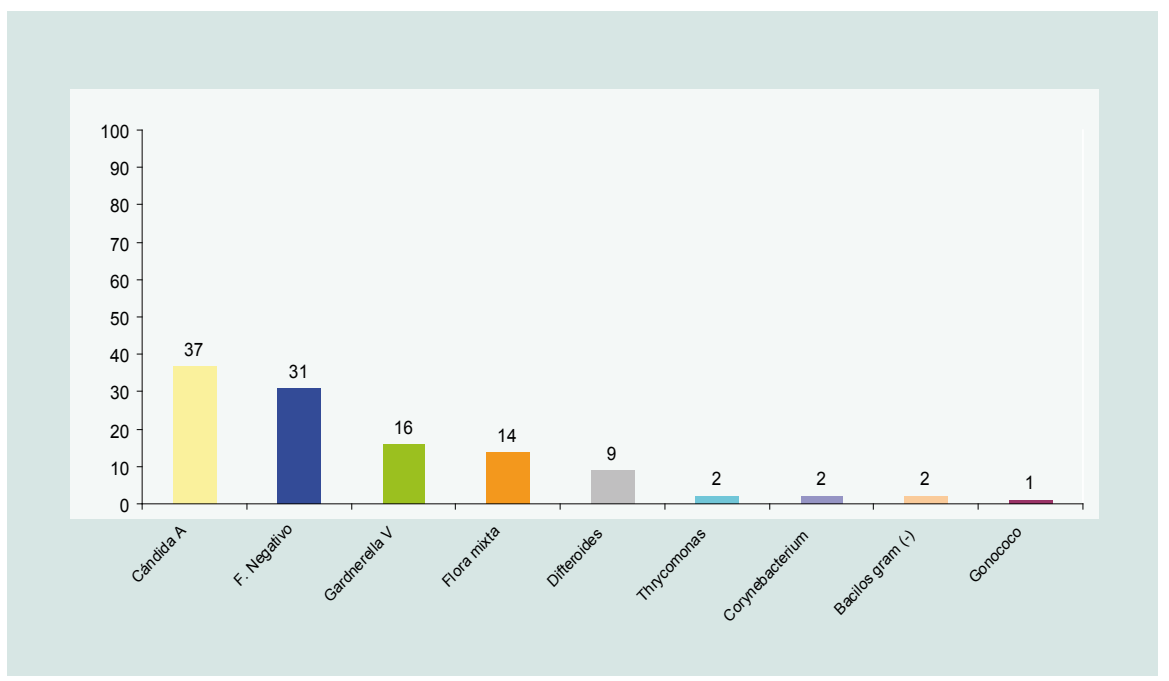

reportaron el prurito como de intensidad severa o moderada al momento de ingreso. Al segundo día de utilización de la solución de bardana y aún sin iniciar tratamiento antibiótico específico, tan sólo 22,5\% de la mujeres manifestaron presentar estas dos categorías de severidad. En contraste, al final del tratamiento ninguna lo experimentaba como severo y sólo apenas $10 \%$ lo refería como moderado, con un 90\% de ellas reportando ausencia o sólo sensación mínima del prurito.

De otro lado, 75\% de las pacientes manifestaron excelente tolerabilidad al medicamento, 23\% buena y $2 \%$ regular. Las pocas quejas, provenientes de dos pacientes, hacían referencia a la demora en solucionar el problema por no obtener desaparición total del prurito en el primer día; sólo una mujer experimentó irritación local desde el primer día de uso y algunas declararon que el producto era muy suave.

\section{DISCUSIÓN}

Los resultados observados en el presente estudio muestran que la solución de bardana es activa y bien tolerada en relación a la reducción y control del prurito vulvovaginal. Estos resultados son consistentes con los reportados por el Instituto Alfred Fournier 
de París en el estudio multicéntrico de Jean-Marc Bohbot, ${ }^{7}$ en el cual se empleó la solución de bardana en mujeres con vulvovaginitis de diferentes causas así como en las que de manera concomitante presentaban prurito y eritema, quienes obtuvieron una muy buena eficacia terapéutica, destacándose la rápida mejoría tanto del prurito como del mismo eritema. Estos mismos resultados se observaron también en el presente estudio, así como en los presentados por Berlier ${ }^{8}$ del Servicio de Endocrinología y Diabetología del Hospital Pediátrico Débrousse en Lyon (Francia) sobre cuidados de la higiene vulvoperineal, en los cuales se empleó la solución de bardana al 1,2\% en niñas, logrando demostrar una regresión de la leucorrea, del prurito y del eritema de la vulva en todos los casos.

Por otro lado, la solución de bardana al 1,2\% ha demostrado en diversas investigaciones ${ }^{3,4,7-9}$ la ausencia de efectos secundarios, manifestando una excelente tolerabilidad cutánea y vaginal, autorizándose así su empleo en aplicaciones locales con total seguridad terapéutica. ${ }^{9}$ La actividad farmacológica de la bardana definida por la Agencia Francesa de Medicamentos y Productos Sanitarios (AFSSAP), en su boletín dedicado a los "Medicamentos a Base de Plantas", incluye las siguientes indicaciones para la hoja de la bardana (aplicación local): "tradicionalmente utilizada en aplicación local como tratamiento complementario suavizante y anti-pruriginoso de las afecciones dermatológicas".

\section{CONCLUSIÓN}

Finalmente, se puede considerar que la solución de bardana al 1,2\% surge como una alternativa segura y al parecer eficaz para el manejo del prurito vulvovaginal de diversa etiología. Estas observaciones deberán ser validadas a futuro por medio de un ensayo clínico controlado aleatorizado.

\section{AGRADECIMIENTO}

Los autores expresan que para el presente estudio se contó con el apoyo logístico y farmacéutico de
EuroEtika Ltda., a través del suministro del producto "Saforelle Fem ${ }^{\circledR}$ " (solución de bardana al 1,2\%).

\section{Lista completa de colaboradores}

- Hoover Canaval, M.D. Profesor Asistente, Departamento de Ginecología y Obstetricia, Facultad de Salud, Universidad del Valle. Hospital Universitario del Valle “Evaristo García” E.S.E. Calle $5^{\mathrm{a}} \mathrm{N}^{\circ} 36-08$, piso $6^{\circ}$. Cali (Colombia). Correo electrónico: hcanaval@univalle.edu.co

- Jairo Bonilla, M.D. Profesor del Departamento de Ginecología y Obstetricia, Fundación Universitaria de Ciencias de la Salud. Bogotá (Colombia).

- Claudia Scarpetta M.D. Médico Gineco-obstetra. Cali (Colombia).

- Fernando Martínez, M.D. Médico Gineco-obstetra. Bogotá (Colombia).

- Jaime Orejarena, M.D. Médico Gineco-obstetra. Bogotá (Colombia).

- Diana Solórzano, M.D. Médico Gineco-obstetra. Cali (Colombia).

- Roberto Gallo, M.D. Médico Gineco-obstetra. Bogotá (Colombia).

- Alberto Hincapié, M.D. Médico Gineco-obstetra. Cali (Colombia).

- María Leonor Fonnegra, M.D. Médico Gineco-obstetra. Bogotá (Colombia).

- Carlos Vallejo, M.D. Médico Gineco-obstetra. Sincelejo (Colombia).

- Jorge Rugeles, M.D. Médico Gineco-obstetra. Valledupar (Colombia).

- Claudia Santarelli, M.D. Médico Gineco-obstetra. Bucaramanga (Colombia).

- María Mercedes Castillo, M.D. Médico Gineco-obstetra. Bogotá (Colombia).

- Carlos Gómez, M.D. Médico Gineco-obstetra. Cúcuta (Colombia).

- Álvaro Linero, M.D. Médico Gineco-obstetra. Santa Marta (Colombia).

- Jorge Tirado, M.D. Médico Gineco-obstetra. Medellín (Colombia).

- Clara H. Torres, M.D. Médico Gineco-obstetra. Cali (Colombia).

- Antonio Manrique, M.D. Médico Gineco-obstetra. Armenia (Colombia).

- Luis Felipe Estrada, M.D. Médico Gineco-obstetra. Cali (Colombia). 
- Manuel Amat, M.D. Médico Gineco-obstetra. Barranquilla (Colombia).

- Adriana Trujillo, M.D. Médico Gineco-obstetra. Ibagué (Colombia).

- Sonia Salamanca, M.D. Médico Gineco-obstetra. Bogotá (Colombia).

- Francisco Vergara, M.D. Médico Gineco-obstetra. Bogotá (Colombia).

- María Cecilia Arturo, M.D. Profesora, Facultad de Medicina, Universidad Libre de Cali. Cali (Colombia).

- Mario Julio Mendoza, M.D. Profesor, Facultad de Medicina, Universidad San Martín. Barranquilla (Colombia).

- Orlando Borré, M.D. Profesor, Facultad de Medicina, Universidad de Cartagena. Cartagena (Colombia).

- Gustavo Gómez, M.D. Profesor, Facultad de Salud, Universidad Surcolombiana. Neiva (Colombia).

- José León, M.D. Profesor, Facultad Ciencias de la Salud, Universidad Tecnológica de Pereira. Pereira (Colombia).

\section{REFERENCIAS}

1. Rees JL, Laidlaw A. Pruritus: more scratch than itch. Clin Exp Dermatol 1999;24:490-3
2. Krajnik M, Zylicz Z . Pruritus in advanced internal diseases. Pathogenesis and treatment. Neth J Med 2001;58:27-40

3. Anderson MR, Klink K, Cohrssen A. Evaluation of vaginal complaints. JAMA 2004;291:1368-79.

4. Lin CC, Lu JM, Yang JJ, Chuang SC, Ujiie T. Antiinflammatory and radical scavenge effects of Arctium lappa. Am J Chin Med 1996;24:127-37.

5. Rodríguez P, Blanco J, Juste S, Garcés M, Pérez $\mathrm{R}$, Alonso L, et al. Allergic contact dermatitis due to burdock (Arctium lappa). Contact Dermatitis 1995;33:134-5.

6. Hanna NF, Taylor-Robinson D, Kalodiki-Karamanoli M, Harris JR, McFadyen IR. The relation between vaginal $\mathrm{pH}$ and the microbiological status in vaginitis. Br J Obstet Gynecol 1985;92:1267-71.

7. Bohbot JM, Ecossistema vaginal e produtos de higiene íntima. Mundo Médico; 2005. Visitado en 2010 Mar 3. Disponible en: http://www.jasfarma.pt/publicacao.php?publicacao $=$ mm\&numero $=43$

8. Berlier P. Soins d'hygiène vulvopérinéale. Médecine \& enfance 1997;17:105-7.

9. Cope R. Interest of Saforelle cream for anal pruritus treatment. Médicine et chirurgie digestives 1999; 28:215-6. 